\title{
Declaración de Panamá: contribuyendo a reducir la mortalidad en hemodiálisis en Centroamérica y el Caribe
}

\author{
Declaration of Panama: Contributing to reduce mortality on haemodialysis in Central \\ America and the Caribbean
}

\author{
Guillermo Álvarez-Estévez' ${ }^{1}$ Ramón García-Trabanino2,3*, Régulo Valdés-Miranda4, \\ Jorge Pérez-Oliva-Díaz ${ }^{5}$, Julio E. Silva ${ }^{6}$, Vicente Sánchez-Polo,8, Gaspar Rodríguez ${ }^{9}$, Nubia Cano ${ }^{10,11}$, \\ Néstor Ayala-Gómez ${ }^{12}$ y Claudio Mascheroni ${ }^{13,14}$
}

${ }^{1}$ Centro de Diagnóstico, Medicina avanzada, Conferencias Médicas y Telemedicina CEDIMAT, Santo Domingo, República Dominicana; ${ }^{2}$ Centro de Hemodiálisis, San Salvador, El Salvador; ${ }^{3}$ Fondo Social de Emergencia para la Salud, Cantón Tierra Blanca, Usulután, El Salvador; ${ }^{4}$ Caja de Seguro Social de Panamá, Panamá, Panamá; ${ }^{5}$ Instituto Nacional de Nefrología Dr. Abelardo Buch López, La Habana, Cuba; ${ }^{6}$ Medicina Corporativa de Diálisis, Guatemala, Guatemala; ${ }^{7}$ Servicio de Nefrología y Trasplante Renal, Instituto Guatemalteco de Seguridad Social, Guatemala, Guatemala; ${ }^{8}$ Programa de Formación de Nefrólogos, Universidad de San Carlos e Instituto Guatemalteco de Seguridad Social, Guatemala, Guatemala; ${ }^{9}$ Unidad de Hemodiálisis, Instituto Hondureño de Seguridad Social, Tegucigalpa, Honduras; ${ }^{10}$ Servicio de Nefrología, Hospital Escuela Antonio L. Fonseca, Managua, Nicaragua; ${ }^{11}$ Servicio de Hemodiálisis, Hospital Salud Integral, Managua, Nicaragua; ${ }^{12}$ Clínica de Hemodiálisis, San Salvador, El Salvador; ${ }^{13}$ Servicio de Nefrología San Pedro, San Pedro, Argentina; ${ }^{14}$ Universidad Nacional de Rosario, Rosario, Argentina

La enfermedad renal crónica (ERC) constituye un gran problema de salud que afecta cerca del $10 \%$ de la población mundial. De acuerdo con la Organización Mundial de la Salud, la Organización Panamericana de Salud (OPS), la Sociedad Internacional de Nefrología y la Sociedad Latinoamericana de Nefrología e Hipertensión (SLANH), la ERC se considera una pandemia de alta relevancia en constante aumento. En la región de Centroamérica y el Caribe, el número de pacientes con ERC crece vertiginosamente debido a la epidemia global de diabetes, hipertensión arterial y obesidad. A estas causas, se suma una nueva forma de ERC de origen aún no claramente determinado, que es altamente prevalente en toda el área costera del océano Pacífico, desde el sur de México hasta Panamá, conocida como nefropatía mesoamericana. En 2014, la OPS y la SLANH acordaron estimular los países centroamericanos y del Caribe a lograr alcanzar el acceso universal a las terapias de reemplazo renal (TRR). Se propusieron alcanzar una prevalencia de al menos 700 pacientes por millón de población (pmp) en TRR para el año 2019. Las estrategias principales para alcanzar este objetivo han sido el desarrollo de registros oficiales de diálisis y trasplante en cada uno de los países, así como fomentar la disponibilidad universal de la TRR costo-efectivas, estrategias en las que se han obtenido importantes avances en algunos de los países de la región.

Hacia el año 2017, en el marco de la Reunión Centroamericana y del Caribe de Diálisis Peritoneal, organizada por la Región 4 de la SLANH y la Asociación Centroamericana y del Caribe de Nefrología e Hipertensión (ACECANH), se estimó que la población en TRR para dicho año en los países de Centroamérica, Cuba y República Dominicana era de 27,170 pacientes: $18,020(66.3 \%)$ en hemodiálisis y $9,150(33.7 \%)$ en diálisis peritoneal, aunque con diferencias notables entre países en cuanto a las tasas de prevalencia. Esta

\section{Correspondencia:}

*Ramón García Trabanino

E-mail: rgt@anhaes.org
Fecha de recepción: 13-09-2018

Fecha de aceptación: 03-10-2018

DOI: 10.24875/NEFRO. 18000049
Disponible en internet: 09-08-2019 Nefro Latinoam. 2019;16:10-12 www.nefrologialatinoamericana.com Access bajo la licencia CC BY-NC-ND (http://creativecommons.org/licenses/by-nc-nd/4.0/). 
prevalencia regional de pacientes en TRR dialítica era de 392.2 pacientes pmp, siendo la hemodiálisis la de mayor prevalencia con $260.1 \mathrm{pmp}$, aunque este dato no refleja la cantidad o la calidad de hemodiálisis que los pacientes reciben, que en muchos casos no es la adecuada.

Con el objetivo de conocer la realidad de la hemodiálisis en Centroamérica y el Caribe, la SLANH, a través de su vicepresidencia regional, y en conjunto con la ACECANH, organizó la primera Reunión Centroamericana y del Caribe de Hemodiálisis, que se realizó del 22 al 25 de febrero de 2018 en la ciudad de Panamá. El lema de esta actividad fue «Disminuir la mortalidad en hemodiálisis».

En ese sentido, se diseñó una encuesta que contenía variables básicas sobre la práctica de la hemodiálisis que fue circulada entre las distintas sociedades nacionales de nefrología de cada uno de los países de Centroamérica, Cuba y la República Dominicana.

Reunidos los presidentes de las sociedades nacionales, los delegados nefrólogos expertos de cada sociedad, el comité de la SLANH de hemodiálisis y los presidentes de la ACECANH -el vicepresidente de la Región 4 de la SLANH y el presidente de la SLANHse llegó a importantes conclusiones y se decidió comunicar las siguientes resoluciones y recomendaciones relativas a los países de Centroamérica y el Caribe:

Generales:

- Se acepta como regla universal sobre los servicios de TRR el derecho del paciente a seleccionar su tipo de terapia de diálisis.

- Se reconoce el derecho universal que tienen los pacientes a recibir el tratamiento de diálisis bajo los parámetros modernos de calidad mínima y se solicita a las autoridades sanitarias de los países que aumenten la disponibilidad de los servicios de diálisis a todos los pacientes que lo requieran.

- Se reconoce que actualmente no hay disponibilidad suficiente de hemodiálisis en ciertos países de Centroamérica, como son Costa Rica, Nicaragua y El Salvador.

- Se recomienda elaborar protocolos y guías nacionales y regionales de hemodiálisis, además de incentivar la formación y especialización en hemodiálisis del personal de enfermería.

Específicas:

- Unidades de hemodiálisis

- De forma inadecuada, actualmente el $37 \%$ de las unidades de hemodiálisis son atendidas por médicos no nefrólogos, lo que indica que el número de nefrólogos es insuficiente o que estos están mal distribuidos.

- Se debe estimular que las unidades de hemodiálisis cuenten con el apoyo de los servicios indispensables de nutrición, psicología, cirugía vascular y trabajo social.

- Es obligatorio cumplir con las recomendaciones de los esquemas de vacunación para los pacientes en hemodiálisis.

- Las autoridades y los ministerios de salud deben legislar para asegurar que en todos los servicios de hemodiálisis, ya sean estatales o contratados, se incentive la aplicación de los indicadores de calidad de hemodiálisis modernos, con el apoyo de las respectivas sociedades nacionales de nefrología.

- Se recomienda elaborar una normativa guiada por la sociedad nacional de nefrología de cada país sobre los requerimientos básicos para habilitar y mantener en funcionamiento una unidad de hemodiálisis.

- Accesos vasculares:

- Se recomienda impulsar el desarrollo de la nefrología intervencionista e incluir la formación en esta disciplina en los sistemas de formación en nefrología.

- Se debe fomentar que la primera opción de acceso vascular sea la fístula arteriovenosa (FAV). El uso de catéteres tunelizados permanentes debe ser la alternativa cuando el paciente no disponga de terreno vascular viable para la FAV.

- Se debe incentivar la creación de clínicas de accesos vasculares a disposición de las unidades de hemodiálisis, y las normas de accesos vasculares deben estar disponibles en todas las unidades de hemodiálisis.

- Las unidades de hemodiálisis deben contar idealmente con un área adecuada para colocar catéteres.

- Registros

- Con el lema «Información para la acción» se recomienda a los ministerios de salud de cada país adoptar como alta prioridad la creación y el desarrollo de registros nacionales de diálisis y trasplante, con el objetivo de poder generar información de calidad que permita conocer la realidad de las TRR en cada país y en la región, así como su impacto sobre la historia natural de la enfermedad. Ello constituye una demanda y prioridad indispensable según la OPS.

- Se reportan inicios en la conformación de los registros en la mayoría de los países. Se deben priorizar las acciones para incentivar y reforzar los 
registros en El Salvador, Honduras, Nicaragua y Costa Rica.

- Es indispensable que las sociedades nacionales sean parte integral de estos registros.

- Sesiones de hemodiálisis:

- Es imprescindible respetar las reglas universales de las sesiones de hemodiálisis: cada paciente debe tener acceso a recibirla tres veces por semana y, en general, de una duración de 4 horas cada sesión.

- Se recomienda aplicar los requerimientos básicos y los indicadores de calidad en hemodiálisis que permitan satisfacer el derecho inalienable de los pacientes a la vida, asegurando su supervivencia en hemodiálisis y, como consecuencia, una disminución de la mortalidad por ERC.

- Suministro de medicamentos:

- Actualmente, la mayoría de los gobiernos de la región no están suministrando adecuadamente los insumos y medicamentos necesarios para el manejo integral del paciente en diálisis, que deben incluir: 1) adecuado control de la hipertensión arterial, 2) adecuado control de la anemia y 3) adecuado control del metabolismo óseo y mineral, todo según las guías internacionales.

- Se recomienda que las autoridades y los ministerios de salud de todos los países de la zona provean la cobertura completa y universal de todos los medicamentos, insumos y tecnologías sanitarias relacionados con el tratamiento de diálisis, a fin de lograr una mejor calidad de tratamiento y así mejorar la calidad y cantidad de vida del paciente en TRR.

Dada y firmada en la ciudad de Panamá, Panamá, a los 25 días del mes de febrero del año dos mil dieciocho.

Firmantes de la declaración:

Dr. Alfonso M. Cueto Manzano. Presidente de la Sociedad Latinoamericana de Nefrología e Hipertensión SLANH, México.

Dr. Guillermo Álvarez Estévez. Vicepresidente Región 4 SLANH y Presidente Asociación Centroamericana y del Caribe de Nefrología e Hipertensión ACECANH, República Dominicana.
Dr. Claudio Mascheroni. Coordinador Comité SLANH de hemodiálisis, Argentina.

Dr. Régulo Valdés Miranda. Presidente Sociedad Panameña de Nefrología e Hipertensión, Panamá.

Dr. Jerónimo Centeno. Coordinador Comité SLANH de hemodiálisis, Brasil.

Dr. Ramón García Trabanino. Presidente Asociación de Nefrología e Hipertensión Arterial de El Salvador ANHAES y Comité SLANH de hemodiálisis, El Salvador.

Dra. Sandra Rodríguez. Consejera Región 4 SLANH, República Dominicana.

Dr. Julio Silva. Presidente Asociación Guatemalteca de Nefrología, Guatemala.

Dr. Gaspar Rodríguez. Presidente Asociación Hondureña de Nefrología y Trasplante, Honduras.

Dr. Jorge Pérez-Oliva Díaz. Presidente Sociedad Cubana de Nefrología, Cuba.

Dr. Vicente Sánchez Polo. Asociación Guatemalteca de Nefrología, Guatemala.

Dra. Nubia Cano. Asociación Nicaragüense de $\mathrm{Ne}$ frología, Nicaragua.

Dra. Viviana Navarro. Asociación Costarricense de Nefrología, costa Rica.

Dra. Wanda Rodríguez. Sociedad Dominicana de Nefrología, República Dominicana.

Dr. Néstor Ayala Gómez. ANHAES, El Salvador.

\section{Conflicto de intereses}

Julio E. Silva M. es gerente médico de la Terapia de Hemodiálisis de Medicina Corporativa de Diálisis, S.A., filial de Baxter RTS. Los demás autores declaran no tener conflictos de interés.

\section{Financiamiento}

Los autores no recibieron patrocinio para generar la declaración ni para escribir este artículo. La reunión regional de hemodiálisis fue apoyada por Fresenius Medical Care. Sin embargo, el patrocinador de la reunión no tuvo ninguna influencia en el contenido de la reunión, en la redacción de esta declaración, ni tuvo acceso a su contenido antes de su publicación. 